\title{
Implantação de Melhoria de Processos em um Setor de Produção de Software de uma Universidade Federal
}

\author{
Fabiana Freitas Mendes ${ }^{1}$, Hugo Alexandre Dantas do Nascimento ${ }^{1,2}$, Patrícia \\ Gomes Fernandes ${ }^{1}$, Rosângela da Silva Nunes ${ }^{2}$, Caroline da Cunha Mota ${ }^{2}$ \\ 'Instituto de Informática - Universidade Federal de Goiás (UFG) \\ Caixa Postal 131 - 74.001-970 - Goiânia- GO - Brazil
}
${ }^{2}$ Centro de Recursos Computacionais- Universidade Federal de Goiás (UFG)
Caixa Postal 131 - 74.001-970 - Goiânia- GO - Brazil
\{fabianafreitas, hadn, patriciagomes\}@inf.ufg.br, \{rdsnunes, carolinecmota\} @gmail.com.br

\begin{abstract}
This paper describes how an initiative to improve software development processes has been led in a software engineering sector of a Federal Institute of Higher Education. Moreover, it presents the lessons learned during this work. Such lessons, as well as the results of a survey on the degree of implementation of the current project, are serving as a basis for planning the next round of process improvements.
\end{abstract}

Resumo. O presente documento descreve uma iniciativa de melhorias de processos de desenvolvimento de software em um órgão de produção de uma Instituição Federal de Ensino Superior. Além disso, são apresentadas lições aprendidas durante a execução do projeto. Estas liçoes, bem como o resultado de uma pesquisa sobre o grau de institucionalização do processo, estão servindo de base para o planejamento do próximo ciclo de melhorias.

\section{Introdução}

Processo é um conjunto de tarefas inter-relacionadas que transformam entradas em saídas [ISO, 2008]. Em empresas de software, estes processos transformam as ideias ou requisições (entradas) dos clientes em código executável (saída). Muitos são os processos relacionados à construção de software como, por exemplo, o processo de análise de requisitos, o processo de design de software e o processo de gerência de projeto de software, dentre outros.

Baseados na hipótese de que a qualidade do processo de software influencia a qualidade dos produtos [TYRRELL, 2000], muitas organizações têm investido em melhoria de processos de software. Um projeto de melhoria de processos de software tem como objetivo conhecer, definir e/ou melhorar os processos relacionados ao desenvolvimento de software para torná-los mais efetivos e eficientes.

Dentre essas organizações, estão as Instituições Federais de Ensino Superior (IFES), as quais distinguem-se bastante de organizações privadas. A primeira grande diferença está na dificuldade de contratação de novos profissionais, a qual, geralmente, depende da disponibilidade de códigos de vaga criados e autorizados pelo Governo Federal. Outro fator diferencial, é a existência de leis e normais mais rigorosas para contratação de serviços na área de TI, como é o caso da Instrução Normativa No. 4 [BRASIL, 2008]. Além disso, apesar da estabilidade de cargo, não há um plano de carreira específico nas IFES para a área de TI, o que faz com que bons profissionais se sintam desestimulados e migrem para a iniciativa privada, ou para outras instituições públicas que oferecem melhores salários. Esses são alguns dos fatores que interferem no gerenciamento de pessoas nas IFES, tornando tal processo diferente daquele realizado por uma empresa privada. 
Por outro lado, as IFES têm crescido de forma significativa e, consequentemente, demandado uma gama maior de sistemas informatizados que apoiem os seus processos organizacionais. Uma pesquisa recente realizada pelo CGTIC/ANDIFES [CGTIC, 2009] mostrou que várias IFES vêm atuando intensamente no desenvolvimento de sistemas informatizados para atender suas demandas.

Neste contexto, a necessidade por um projeto de melhoria de processos de software é grande em instituições federais de ensino superior que, como a Universidade Federal de Goiás (UFG), possuem um setor de desenvolvimento de software. Todavia, apesar da importância do processo formal, até o presente momento, apenas a Universidade Federal do Pará e a Universidade Federal do Rio de Janeiro possuem unidades de desenvolvimento de software oficialmente qualificadas em algum nível do MPS.BR [SOFTEX, 2010].

O presente trabalho apresenta, assim, a experiência de implementação de melhoria de processos no Centro de Recursos Computacionais (CERCOMP) da UFG, órgão responsável pelo desenvolvimento e manutenção de software dessa instituição.

O restante do artigo está organizado como segue: a Seção 2 apresenta questões relacionadas à melhoria de processos de software; a Seção 3 apresenta os ciclos de melhoria que já foram implementados, as lições aprendidas durante esses ciclos e os próximos passos que estão planejados; finalmente, a Seção 4 apresenta as considerações finais.

\section{Iniciativas de Melhoria de Processos de Software}

As iniciativas de melhoria de processos de software têm utilizado um conjunto de modelos de maturidade e normas (dentre outros elementos) que fornecem diretrizes sobre o quê melhorar, como melhorar e como manter as melhorias dos processos [KIRWAN et al., 2008].

Por exemplo, modelos como o CMMI (Capability Maturity Model Integration) [SEI, 2006] e o MR-MPS (Modelo de Referência do MPS.BR) [SOFTEX, 2009], assim como normas como a ISO 12.207 [ISO, 2008], são fontes de boas práticas, representadas por práticas genéricas, práticas específicas e por resultados esperados de processos e de atributos de processos, que podem ser incorporadas aos processos de software das organizações. Em particular, o MR-MPS e o CMMI propõem uma estrutura de níveis de maturidade, como uma forma de implementação das diferentes áreas de processos (ou simplesmente processos, como denomina o MPS.BR). Como exemplo, o nível inicial do MPS.BR é o nível $G$, o qual engloba os processos de Gerência de Projetos e de Gerência de Requisitos.

Além disso, modelos como o IDEAL (Initiating, Diagnosing, Establishing, Acting, Leveraging) [McFEELEY, 1996] são fontes de práticas para a própria iniciativa de melhoria. Ou seja, estabelecem recomendações sobre como conduzi-la. Tal modelo propõe cinco grande fases para as iniciativas de melhoria, sendo que cada uma é composta de um conjunto de atividades.

A próxima Seção apresenta os ciclos de melhoria já conduzidos no CERCOMP, os quais, conforme será visto, estão sendo executados de acordo com as fases propostas pelo método de implantação de melhorias do IDEAL.

\section{Elaboração do Processos de Software}

O CERCOMP é um órgão da UFG vinculado à PRODIRH (Pró-Reitoria de Desenvolvimento Institucional e Recursos Humanos) responsável pela infraestrutura de TI da universidade, conforme a Resolução CONSUNI No 32/2008 [CONSUNI, 2008]. O CERCOMP possui três divisões - Sistemas, Redes e Suporte, sendo que a Divisão de Sistemas é a que realiza o desenvolvimento e a manutenção dos sistemas que atendem à 
UFG, possuindo atualmente um portfólio de 42 sistemas mantidos por uma equipe de 29 pessoas. Como a quantidade de pessoas é muito pequena em relação à quantidade e à complexidade dos sistemas, as atividades de desenvolvimento somente podem ser executadas a contento com um processo formal que auxilie na utilização dos recursos disponíveis e na qualidade dos sistemas gerados.

Em setembro de 2007, iniciou-se o projeto de melhoria de processo de software no CERCOMP com a criação do Grupo de Processos de Software (GPS), o qual conduz as atividades desse projeto. Foram realizados pelo grupo dois ciclos de melhoria, sendo que um terceiro ciclo está em planejamento.

O restante desta seção descreve o modo de condução dos ciclos já finalizados e as lições aprendidas nos mesmos.

\subsection{Primeiro Ciclo de Melhorias}

Durante o primeiro ciclo de melhorias, foram executadas as cinco fases previstas pelo método IDEAL [McFEELEY, 1996]: iniciação, diagnóstico, estabelecimento, ação e aprendizado.

$\mathrm{Na}$ iniciação, foi apresentado o roteiro geral de melhoria, coletadas e examinadas informações iniciais sobre os processos de software da Divisão de Sistemas e elaborado um plano preliminar do projeto, contendo a equipe inicial, as premissas, as restrições e um cronograma para a fase seguinte. Este plano foi aprovado pelo Gerente da Divisão de Sistemas e pelo Diretor do CERCOMP.

$\mathrm{Na}$ fase seguinte, de diagnóstico, foi verificada a aderência dos processos de software da Divisão de Sistemas em relação ao nível F do MPS.BR [SOFTEX, 2009]. Os métodos utilizados para a coleta de informações sobre os processos consistiram de questionários online e entrevistas. As entrevistas tiveram como objetivo esclarecer pontos obscuros nas respostas dos funcionários questionados.

Ao final dessa fase, foi realizada uma reunião com todos os membros da Divisão de Sistemas com o objetivo de apresentar os resultados do diagnóstico os quais foram validados pelos funcionários.

Passou-se, então, para a fase de estabelecimento, na qual foi feito um planejamento com base nos resultados do diagnóstico. Foram, assim, estabelecidos quatro objetivos de melhorias: (1) dar maior visibilidade aos projetos, (2) organizar o modo como as requisições de serviço chegam à Divisão de Sistemas, (3) gerenciar a configuração dos sistemas desenvolvidos e (4) desenvolver e manter o conhecimento sobre os sistemas críticos.

Para o primeiro objetivo, a solução adotada foi a definição de um processo de Gerência de Projeto, de acordo com o que é exigido pelo nível G do MPS.BR. Além disso, foram definidas algumas ações de pré-projeto com o objetivo de dar visibilidade ao Gerente da Divisão de Sistemas dos projetos iniciados

Para atender o segundo objetivo, as ações de pré-projeto foram reunidas em um processo que define a forma como os usuários requisitam novos produtos ou a manutenção dos produtos já existentes. Foi elaborado ainda um processo de Gerência de Requisitos para lidar com os requisitos que chegam durante um projeto.

Um processo de Gerência de Configuração simplificado (apenas os resultados esperados GCO 1 a 6) foi definido para atender o terceiro objetivo. Além disso, foram definidas ações para institucionalizar o uso de uma ferramenta padrão de controle de versões. Todos os processos definidos foram reunidos no Manual de Produção de Software do CERCOMP [GPS, 2009].

Apesar de terem sido planejadas ações para lidar com o quarto objetivo, elas não foram executadas. A ação planejada era a criação de fluxogramas para descrever as 
principais funcionalidades implementadas pelos sistemas críticos.

As soluções definidas para os quatro objetivos foram implementadas em um projeto piloto, o qual foi executado e teve o acompanhamento do GPS em termos de garantia da qualidade e de mentoring.

Ao final da execução dessas ações (final da Fase Ação e início da Fase de Aprendizado), foi realizada uma reunião de marco que teve como objetivo apresentar os resultados alcançados e as lições aprendidas, e indicar as ações a serem realizadas no próximo ciclo de melhorias.

\subsection{Segundo Ciclo de Melhorias}

O segundo ciclo de melhorias teve como insumo os resultados alcançados no primeiro. Esses resultados demonstraram a necessidade de implementar ações voltadas para a institucionalização dos processos até então definidos. Com este objetivo, foram planejadas as seguintes ações:

- Definir e acompanhar cronograma de adoção do processo. Neste caso, foram definidos projetos que o GPS deveria acompanhar, fornecendo ajuda na compreensão do processo e executando a garantia da qualidade dos mesmos. Esta atividade, contudo, não foi completada, pois sempre que eram definidos projetos para serem acompanhados aconteciam impedimentos como mudança na equipe, atraso no início ou prazo apertado para o término do projeto.

- Analisar ferramentas. Foram analisadas ferramentas para apoiar os pontos mais críticos do processo, ou seja: a manutenção do cronograma do projeto e a manutenção da matriz de rastreabilidade. Entre as ferramentas analisadas, apenas a ferramenta padrão para cronogramação foi definida.

- Definir a forma de trabalho do SAU (Sistema de Atendimento ao Usuário) e acompanhar suas atividades. A definição foi realizada, entretanto, não foi feito o acompanhamento das atividades.

Além destas ações, o manual foi alterado de acordo com as requisições dos colaboradores do desenvolvimento. A principal alteração feita foi a divisão do processo Solução Técnica, que englobava as atividades de análise, projeto (design) e solução técnica e era muito genérico, em processos mais específicos de Análise e de Solução Técnica. Este último, após as alterações, passou a conter atividades relacionadas ao projeto (design), implementação e teste de software.

Ao final deste ciclo de melhorias, foi realizada uma pesquisa com o intuito de avaliar a percepção dos colaboradores da Divisão de Sistemas, coletar opiniões sobre o Processo de Software e identificar os problemas e as dificuldades encontrados.

Foram feitas entrevistas estruturadas, cujas questões estão descritas em [GPS, 2010], com 23 dos 29 funcionários da Divisão de Sistemas, o Diretor do CERCOMP e o gerente do SAU. As entrevistas duraram entre trinta minutos a uma hora e meia. Ao final, foi elaborado um relatório que contém os resultados obtidos [GPS, 2010]. Estes resultados também foram apresentados em uma reunião para todos os funcionários da Divisão de Sistemas e estão sendo utilizados para planejar o terceiro ciclo de melhorias.

\subsection{Lições Aprendidas}

A experiência vivida no CERCOMP nestes quase dois anos e meio gerou diversas lições aprendidas, as quais são apresentadas a seguir, com algumas discussões:

1. Repassar uma mensagem uniforme sobre a iniciativa de melhoria. Todos os membros do GPS e os gerentes superiores devem ter a mesma visão sobre a iniciativa de melhoria. 
2. Começar a iniciativa convencendo os gerentes superiores sobre os recursos necessários para as melhorias e sobre seus benefícios. Isto seria um trabalho realizado antes do planejamento e depois do diagnóstico de processos. Deveriam ser mostradas alternativas e custos relacionados às melhorias. Como isto não foi feito na iniciativa do CERCOMP, não houve alocação significativa de recursos humanos para a iniciativa de melhoria e o processo definido não ficou adequado à realidade de pessoal.

3. Incluir membros influentes e favoráveis às melhorias ao GPS. No caso do CERCOMP, alguns dos servidores mais antigos eram considerados mais resistentes à adoção do processo. Durante uma pesquisa sobre a institucionalização do processo foi percebido que a inclusão daqueles que eram mais antigos e eram favoráveis à iniciativa poderia ajudar na minimização de resistências dos demais [GPS, 2010].

4. Garantir que todos os envolvidos entendam os objetivos de melhoria e que estes estejam alinhados aos objetivos da organização. No caso do CERCOMP, não ficou claro para todos os colaboradores se o objetivo da melhoria era a qualidade do processo ou a criação de documentação para os sistemas. Isto gerou resistência da parte dos colaboradores.

5. Realizar um diagnóstico com o objetivo de conhecer melhor as equipes, produtos e metas da organização, mais do que simplesmente com o objetivo de verificar a aderência em relação a um modelo de qualidade. Com o passar do tempo na iniciativa, viu-se a necessidade de mudar não apenas o processo de software, mas também a estrutura das equipes. Para isto, era necessário conhecer melhor o contexto do CERCOMP, em termos de produtos (e suas demandas) e pessoas.

6. Planejar e executar ações inclusivas, garantindo o envolvimento e participação de todos os membros que serão afetados com a iniciativa de melhoria. Conforme a iniciativa era conduzida pelo GPS, os demais membros da organização declararam, durante a pesquisa sobre a institucionalização do processo, que sentiam um afastamento entre o GPS e os membros da Divisão de Sistemas. De fato, foi percebido que as pessoas que se envolveram com o projeto piloto foram as que mais se aproximaram espontaneamente do GPS, o que contribuiu para que aumentassem seus conhecimentos sobre o processo e o adotassem. Enquanto isto, o índice de uso do processo entre os demais colaboradores continuou baixo. Desta forma, é preciso envolver no projeto de melhoria as pessoas diretamente associadas aos processos que estão sendo definidos.

7. Evitar ciclos de melhorias longos e definir pequenos alvos de melhoria. Incluir, desde o início, ações de institucionalização e um acompanhamento rígido sobre todas as atividades executadas. É preferível também definir apenas um processo ou uma boa prática e trabalhar para sua institucionalização, do que definir uma quantidade grande de processos e depois institucionalizá-los. Durante a iniciativa foi percebido que os colaboradores já adotavam esta postura naturalmente, pois os processos técnicos (Análise e Solução Técnica) estavam sendo mais amplamente utilizados do que os processos gerenciais (Gerência de Projetos, Gerência de Requisitos e Gerência de Configuração) [GPS, 2010].

8. Priorizar a análise de ferramentas para o apoio aos processos definidos. Um dos problemas identificados sobre a não institucionalização do processo de software durante a pesquisa realizada estava relacionado à falta de ferramentas de apoio ao processo [GPS, 2010]. Desta forma, é altamente recomendável que se defina o processo de software e, ao mesmo tempo, já se determine ferramentas padrão que o apoiem, mesmo que o processo tenha que sofrer pequenos ajustes para ser totalmente auxiliado pela ferramenta. Também é 
importante analisar criteriosamente tais ferramentas.

9. Ministrar treinamentos frequentes durante toda a iniciativa de melhoria. Esses treinamentos deveriam estar focados mais no "como fazer" do que em "o que fazer". Além disso, deveriam existir treinamentos para incutir conceitos gerais de engenharia de software nos colaboradores que utilizarão o processo. Também devem ser feitos treinamentos nas ferramentas definidas como padrão. Se necessário, deve-se criar um roteiro de como utilizar a ferramenta como apoio ao processo.

10. Constituir uma equipe de garantia da qualidade para ajudar na aplicação do processo. No caso do CERCOMP, o foco inicial eram nos processos do nível $\mathrm{G}$ do MPS.BR, o qual não inclui um processo de Garantia da Qualidade. Porém, foi percebida esta necessidade ao longo da iniciativa. Assim, mesmo que o GPS tenha que assumir algumas responsabilidades de Garantia da Qualidade, é interessante aplicar conceitos de tal processo desde o início da iniciativa.

11. Acompanhar as atividades do GPS de forma mais ágil. Como o GPS não contava com muitas pessoas durante a maior parte da iniciativa, um trabalho mais ágil, em uma organização em estágios iniciais de maturidade, como no caso do CERCOMP, pode permitir que o grupo assuma um papel mais focado em ajudar as pessoas no seu trabalho com o processo, aumentando o apoio ao mesmo.

12. Definir e registrar inicialmente um processo mais simplificado. Em consonância com a lição de número 7 , esta necessidade foi identificada durante a pesquisa feita sobre a institucionalização do processo de software [GPS, 2010].

13. Respeitar as restrições necessárias para o sucesso de um projeto piloto. No caso do CERCOMP, desde o início, foi colocado que o projeto piloto deveria servir para avaliar apenas o processo de software definido. Porém, ao longo do projeto, o gerente do projeto piloto foi mudado (para um colaborador menos experiente) e a linguagem de programação escolhida para a implementação também era nova para os colaboradores. Isto fez com o que o projeto piloto avaliasse vários itens ao mesmo tempo.

14. Ter gerentes superiores sempre disponíveis para o GPS. O contato constante entre estes dois grupos pode aumentar os conhecimentos dos gerentes superiores sobre o processo contribuindo para sua institucionalização, já que esses gerentes coordenam o trabalho dos demais colaboradores.

15. Ter um membro do GPS dedicado exclusivamente à gerência do projeto de melhorias. Enquanto houve um membro do GPS dedicado à gerência do projeto de melhorias, a iniciativa foi executada conforme o planejado. Porém, quando este membro foi alocado para outras atividades em paralelo, a gerência da iniciativa foi prejudicada.

Estas lições aprendidas estão servindo de base para o planejamento do próximo ciclo de melhorias da iniciativa.

\section{Conclusões}

O presente trabalho mostrou como foram e estão sendo conduzidas as melhorias de processos do órgão responsável pelo desenvolvimento e manutenção de software de uma IFES, sendo este um tipo de experiência pouca divulgada no contexto das universidades federais brasileiras.

Em pouco mais de dois anos de projeto, foram definidos dois ciclos de melhorias. No primeiro deles foi formado o GPS, realizado um diagnóstico das práticas dos processos da Divisão de Sistemas, definido e registrado cinco processos (Pré- 
projeto, Gerência de Projeto, Gerência de Requisitos, Gerência de Configuração, Análise e Solução Técnica) e realizado um teste dos mesmos em um projeto piloto.

No segundo ciclo de melhorias, foram feitos ajustes nos processos definidos anteriormente, analisadas ferramentas de apoio aos processos, e definida a forma de trabalho do SAU. Ao término deste ciclo, foi realizada uma pesquisa com o intuito de verificar o grau de institucionalização dos processos.

Atualmente, o terceiro ciclo de melhorias está sendo planejado com base nos resultados da pesquisa e nas lições aprendidas. Espera-se, como resultado da execução deste ciclo, que os processos tenham o grau de institucionalização esperado. Para tanto, será necessária uma maior interação entre o GPS e os demais membros da Divisão de Sistemas do CERCOMP, e que a estrutura organizacional dessa divisão seja alterada de modo a diminuir o número de equipes com apenas um colaborador.

\section{Referências}

BRASIL. Instrução Normativa No 4 de 19 de Maio de 2008. Ministério do Planejamento, orçamento e gestão. Secretaria de Logística e Tecnologia. Disponível em: $\quad$ http://www.servidor.gov.br/noticias/noticias08/arq down/080519 IN 4.pdf. Acesso em 22 abr. 2010.

CGTI, Colégio de Gestores de TI da ANDIFES. Por um Sistema Integrado para Gestão das IFES. 2008. Disponível em: <forplad.andifes.org.br/apache2default/...2008/sist_int_gestao_cgtic.pdf ->.Acesso em: 12 fev. 2010.

CONSUNI. Conselho Universitário da Universidade Federal de Goiás. RESOLUÇÃ̃ - CONSUNI No 32/2008. Disponível em: $<$ http://www.ufg.br/this2/uploads/files/17/Resolucao_CONSUNI_2008_0032.pdf $>$. Acesso em: 12 de fev. de 2010.

GPS. Grupo de Processos de Software. Relatório da Pesquisa sobre o Processo de Software $\quad$ CERCOMP, 2010. Disponível em $<$ http://portais.ufg.br/this2/uploads/files/17/Relatorio_Pesquisa_Processo_de_Softwa re.pdf $>$. Acesso em: 12 de fev. de 2010.

GPS. Grupo de Processos de Software. Manual de Produção de Software. CERCOMP, 2009. Disponível em <ftp://ftp.ufg.br/Tutoriais/GPS/manual.pdf $>$. Acesso em: 12 de fev. De 2010.

KIRWAN, Pat; SIVIY, Jeannine; MARINO, Lisa; e MARLEY, John. Improvement Technology Classification and Composition in Multimodel Environments. White paper. Disponível em http://www.sei.cmu.edu/library/assets/3.pdf. Mar. 2008.

ISO/IEC, International Organization for Standardization/ International Electrotechnical Comission. ISO/IEC 12207 Systems and software engineering- Software life cycle processes, Geneve: ISO, 2008.

McFEELEY, Bob. IDEAL ${ }^{\text {SM }}$ A User's Guide for Software Process Improvement. CMU/SEI-96-HB-001 Pittsburgh, 1996. 236p.

SOFTEX, Associação Para Promoção da Excelência do Software Brasileiro. Melhoria de Processo de Software Brasileiro (MPS.BR): Guia Geral:2009. Disponível em: $<$ http://www.softex.br/mpsbr/_guias/guias/>. Acesso em: 26 jan. 2010.

SOFTEX, Associação Para Promoção da Excelência do Software Brasileiro. Avaliações MA-MPS.BR. 2010. Disponível em: $<$ http://www.softex.br/mpsbr/_avaliacoes/avaliacaoTodas.asp>. Acesso em: 12 fev. 2010 .

TYRRELL, Sebastián. The many dimensions of the software process. Crossroads. ACM, New York, p. 22-26. jun. 2000. 